\title{
STUDIES WITH RADIOACTIVE DI-AZO DYES. 1. THE LOCALIZA- TION OF RADIOACTIVE DI-BROM TRYPAN BLUE IN INFLAMMATORY LESIONS
}

\author{
BY FRANCIS D. MOORE 1 AND LESTER H. TOBIN \\ (From the Medical Laboratories of the Collis P. Huntington Memorial Hospital, Harvard \\ University, and the Surgical Services of the Massachusetts General Hospital, Boston)
}

(Received for publication February 26, 1942)

The localization of circulating colloidal dyes in areas of increased capillary permeability, and their ingestion there by phagocytic cells, has been under investigation for the past 30 years. First observed by Goldmann (1) during his early studies with the vital stains, it later became the subject of study by investigators working with the inflammatory process, who found that many types of inflammation caused sufficient increase in permeability of the capillaries to produce local accumulations of dye $(2,3,4)$.

Evans (5) described the macrophage system as a separate entity as a result of its delineation by such dyes, following an extensive study of the intra-vitam reactions of many dyes of the benzidine series, such as trypan blue (6). More recently, many workers ( 7 to 10 ) have used dyeaccumulation as an index of the extent of permeability changes following injections of various irritants, fractions of inflammatory exudates, or organ-extracts, in an effort to elucidate the details of the inflammatory sequence. Burrows (11) provides an excellent review of the work in this field prior to 1932.

Keith, Rowntree and Geraghty (12) explored the use of colloidal dyes in blood-volume determinations, this application arising from the fact that many of the acid azo dyes remain intravascular for fairly prolonged periods of time, and appear to form a bond with plasma protein (13). They, and others who followed in the same field (Dawson, Evans and Whipple (14), and Gregerson, Gibson and Stead (15)) have added much to our knowledge of the intravascular reactions of the dyes through their quantitative observations on serum concentrations, rates of elimination from the blood stream, and toxicity. Through this

1 This study was carried out under a Fellowship from the National Research Council.

This is reprint number 557 of the Harvard Cancer Commission. work a new dye came into common use, called Evans blue (so named because its biological properties were first described by Prof. H. M. Evans (14)), otherwise known as T-1824. The latter name is based on the structure of the dye, indicating that tolidine is coupled with the "1-8-2-4-" acid (1-amino-8-naphthol-2, 4-di-sulfonic acid). This dye is isomeric with trypan blue, which by the same type of nomenclature would be "T1836."

In the past five years, work has begun to appear in the literature bearing on the potential clinical application of the property of these dyes of concentrating in abscesses. Menkin (9) saw the potentialities of this field and wrote that trypan blue might be used to enhance the roentgen appearance of abscesses. Strauss, et al. (16) conceived the idea that some substance which would concentrate in abscesses might be made radioactive and so be useful as a means of localizing them clinically. This group has studied various radioactive and non-radioactive substances including a non-radioactive brominated Evans blue, and a radioactive brominated "H-acid" (the 1-8-3-6 sulfonic acid comprising a portion of the trypan blue molecule) $(17,18)$. They did not employ very large doses of radioactivity and their radioactive readings from intact animals were quite low. Work is not reported with an actual radioactive dye; $\mathrm{H}$-acid is neither colloidal nor a dye, although it is a dyeintermediate used in the manufacture of trypan blue. They conclude that the method holds some promise and deserves further work.

A colloidal dye such as trypan blue, by virtue of its property of accumulation in abscesses, offers us a means of diagnosing localized inflammation if we can make such a dye radioactive. The dye must be radioactive to a degree permitting its detection from outside the patient with a Geiger counter. Furthermore, the radioactive element must be firmly affixed to the molecule by a non- 
dissociating bond so that the radioactivity is carried throughout its course in the body by the larger organic molecule. For example, a sodium salt of such a dye made with radioactive sodium would be useless for such a purpose because the sodium is attached to the molecule by a polar bond, and dissociates in solution. Therefore, the radioactive sodium would exchange with stable sodium immediately upon introduction into the circulation and the dye would no longer, in any sense, be radioactive.

Were this diagnostic tool to be perfected, lesions such as appendiceal, sub-diaphragmatic or brain abscesses would become more certain of diagnosis and more susceptible to accurate treatment.

We wish herein to report the study of the localization in inflammatory lesions of a radioactive colloidal di-azo acid dye, di-brom trypan blue.

\section{EXPERIMENTAL PROCEDURE}

\section{Chemistry}

The trypan blue molecule consists of a di-phenyl diamine (ortho-tolidine) coupled through two azo linkages to two equivalents of $\mathrm{H}$-acid (1-amino-8-naphthol-3, 6-disulfonic acid). We have rendered the molecule radioactive by adding two atoms of radio-bromine to the ortho-tolidine, and then coupling it with the acid to make the finished dye. The structural formula of the dye and the position of the bromines is shown in Figure 1.

Radio-bromine is chosen to confer radioactivity on the molecule because bromine is reactive chemically and $\mathrm{Br}^{82}$ has a penetrating gamma-ray. ${ }^{2}$ The half-life of $\mathrm{Br}^{\mathrm{Bz}}$ is 34 hours (19):

The brominated dye is red rather than blue; it is colloidal and will pass through a cellophane membrane only very slowly (4 per cent in 24 hours); it conducts itself in animals substantially as trypan blue does. Because of the identification of the di-brom tolidine, we are assured of the position of the radio-bromines in the molecule. This radioactivity is firmly affixed to the molecule because bromine on an aromatic ring does not ionize or become dissociated under ordinary conditions.

However, adding two bromines to this molecule has

2 The authors wish to express their gratitude to Prof. Robley D. Evans and the Cyclotron Group at the Massachusetts Institute of Technology, and Dr. Baldwin Curtis and the Cyclotron Staff of Harvard University, for providing them with radio-bromine. Dr. Arthur Kip of the Massachusetts Institute of Technology has given the authors much assistance with the physical detection equipment used in this study.

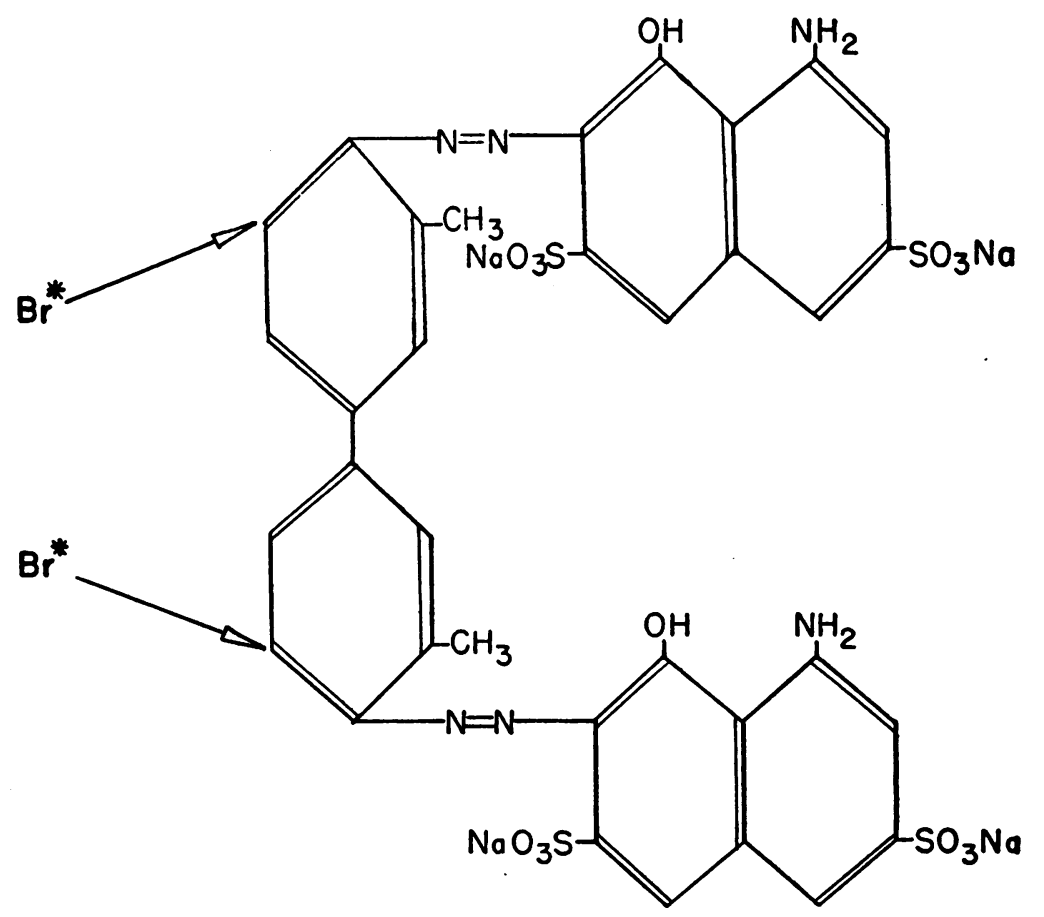

Fig. 1. The Structural formula of Trypan Blue, Showing the Points at which Radioactivity is Conferred upon the Molecule by the Addition of Two Atoms of Radioactive Bromine 
increased the molecular weight by 16.8 per cent and it is to be expected that chemically and biologically the dye may differ somewhat from trypan blue. ${ }^{3}$

\section{Animal techniques}

Our first experiments were done on rats, employing concentrated sterile broth injected intradermally as a means of producing the inflammatory process (9). The animals were given their dye intravenously, coincident with the induction of the inflammation, and two hours later anaesthetized with intraperitoneal Nembutal, and studied with an unscreened Geiger counter.

In an attempt to approximate more closely the conditions present in human inflammation, experiments were then done on rabbits using bacterial abscesses. These lesions were produced by the injection of virulent staphylococci around a mass of agar, which had been injected warm and allowed to gelatinate in the subcutaneous tissues. This gelatinous mass provides a nidus around which the infection may develop. Using such a technique, gross signs of inflammation are evident in 1 to 2 hours; in 6 to 12 hours the area becomes edematous. By 24 to 48 hours the process has either resolved or gone on to form a localized chronic abscess which may rupture and become ulcerated.

The staphylococci were grown on blood agar for 48 hours, with frequent transplants as a means of increasing their virulence. But even with these precautions there was sufficient variation in virulence and animal resistance to become a factor in interpretation of the results.

During the 6 to 12 hour period in the development of the abscess, radioactive di-brom trypan blue was injected intravenously, usually in fractional doses over a period of 4 to 6 hours. An hour after the last dose, the animals were anaesthetized with ether or intramuscular Nembutal and taken to the counter to study the distribution of the radioactive dye.

\section{Dosage and counting technique}

In rats, 0.1 to $0.5 \mu c .4$ was a sufficient dose of radioactive dye to give satisfactory readings. For rabbits, 1.5 to $3.0 \mu \mathrm{c}$. was found to be satisfactory. The dye was used in concentrations of 0.25 per cent to 0.5 per cent, and from 6 to $40 \mathrm{cc}$. of dye were necessary to administer the proper amount of radioactivity, depending on what period of time had elapsed after the standardization measurements

\footnotetext{
${ }^{3}$ Methods of production of free bromine from the cyclotron targets, the synthesis of the dye, its purification and properties are to be described in a subsequent paper (20).

4 The unit $\mu$ c. (micro-curie), as used here, is expressed in reference to a uranium standard. It represents a radioactivity reading equivalent to that given by one microcurie of uranium. This amounts to about $1.6 \times 10^{8}$ counts per minute on our instrument. The bromine isotopes of short half-life are allowed to decay away before making the measurements so that the figures represent activity from $\mathrm{Br}^{82}$ exclusively.
}

were made. It will be recalled that the half-life involved is 34 hours, so that each 34 hours twice as much dye must be used to administer an equivalent amount of radioactivity. On an average, $20 \mathrm{cc}$. of 0.25 per cent dye totalled $2.0 \mu \mathrm{c}$. and made a satisfactory rabbit dose. Dyes of this strength have been useful for animal experimentation for a week after synthesis. The total amount of dye administered (50 to $100 \mathrm{mgm}$.) falls into the range previously used by investigators using trypan blue $(9,10)$.

The detecting instrument is a Geiger counter, screened with lead so as to focus its registration on one portion of the animal at a time. This device and the amount of lead used are shown in Figure 2. The counter and counting-rate meter are connected to a recording milliammeter, so that the number of counts per minute emanating from any portion of an animal is integrated and recorded. This counter has a background of counts from cosmic rays and local radiation of other sources, of about 50 counts per minute (C.P.M.). The background count is subtracted from all the readings taken from the animal to give the net C.P.M. for any specific area. With the doses mentioned, readings from such an area on the body of the rabbit fall into the range of 500 to 2000 C.P.M.

The animal is anaesthetized so that it will lie quietly, and is then moved about under the counter to study the radioactivity in its various portions and in the abscess. The C.P.M. registered from the various points are entered on a standard diagram of the animal to make a permanent record. It can then be determined to what extent the dye has accumulated in the abscess, and therefore to what degree the dye accumulation indicates the location of the lesion.

\section{RESULTS}

\section{In rats}

The findings in the initial experiments on rats are shown in Figure 3. The inflammatory processes were on the abdomen, and roughly 50 per cent more C.P.M. were registered from that region than from the thorax. The control animal, on the other hand, showed fewer C.P.M. over the abdomen than over the thorax. This indicated concentration of the dye in the lesion to an extent detectable with the counter, this finding being correlated with the appearance of dye visually at the site.

\section{Distribution in normal rabbits}

An animal larger than the rat is desirable for this work because it allows the making of a more detailed study of the distribution of the radioactive dye in the intact animal. On the rabbit, 10 or 12 readings may be made down the midline of the abdomen with a counter of this type, 


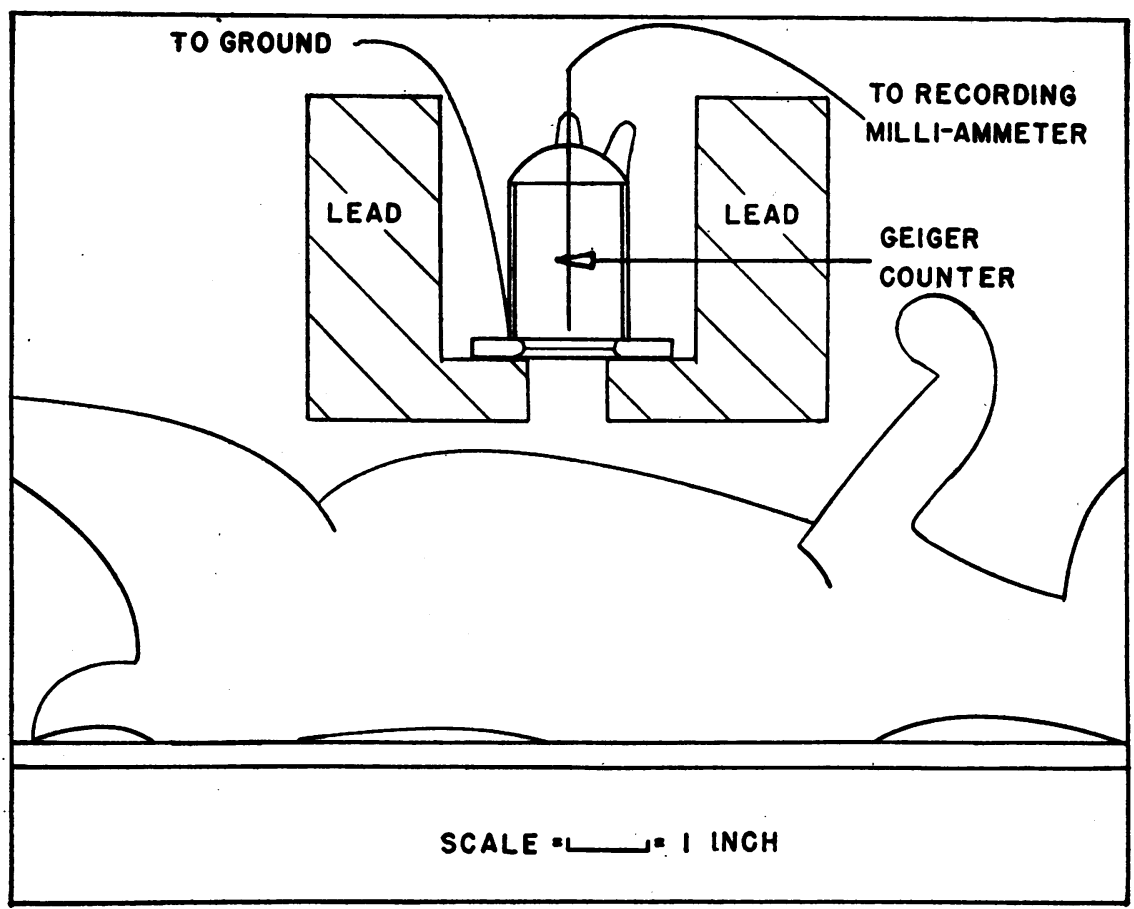

Fig. 2. Cross-section Diagram of Lead Shield Surrounding Geigrr Counter so as to Admit Radiation from Small Portion of an Animal at a Time

The position of the anesthetized animal is also shown.

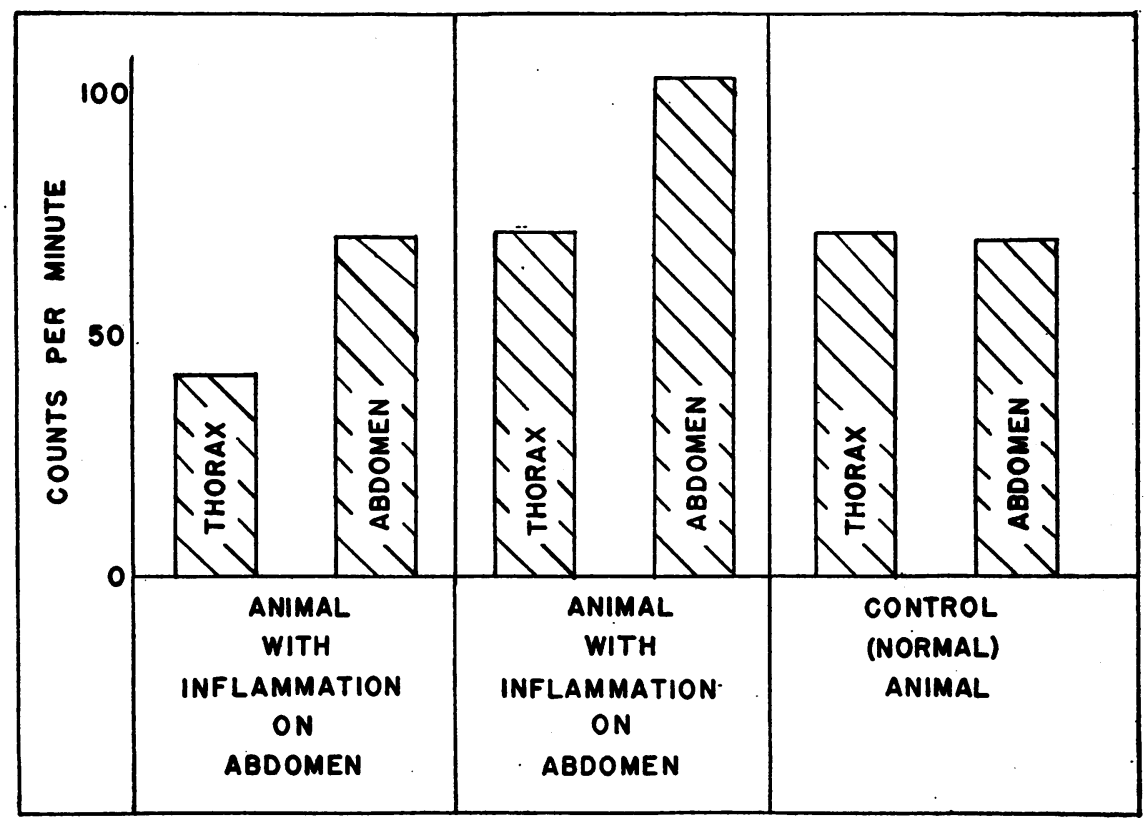

Fig. 3. Chart Showing the Number of Counts per Minute Registered from the Thorax and Abdomen of Two Rats with Inflammation on the Abdomen, in Contrast to the Corresponding Readings from a Normal Control Animal 


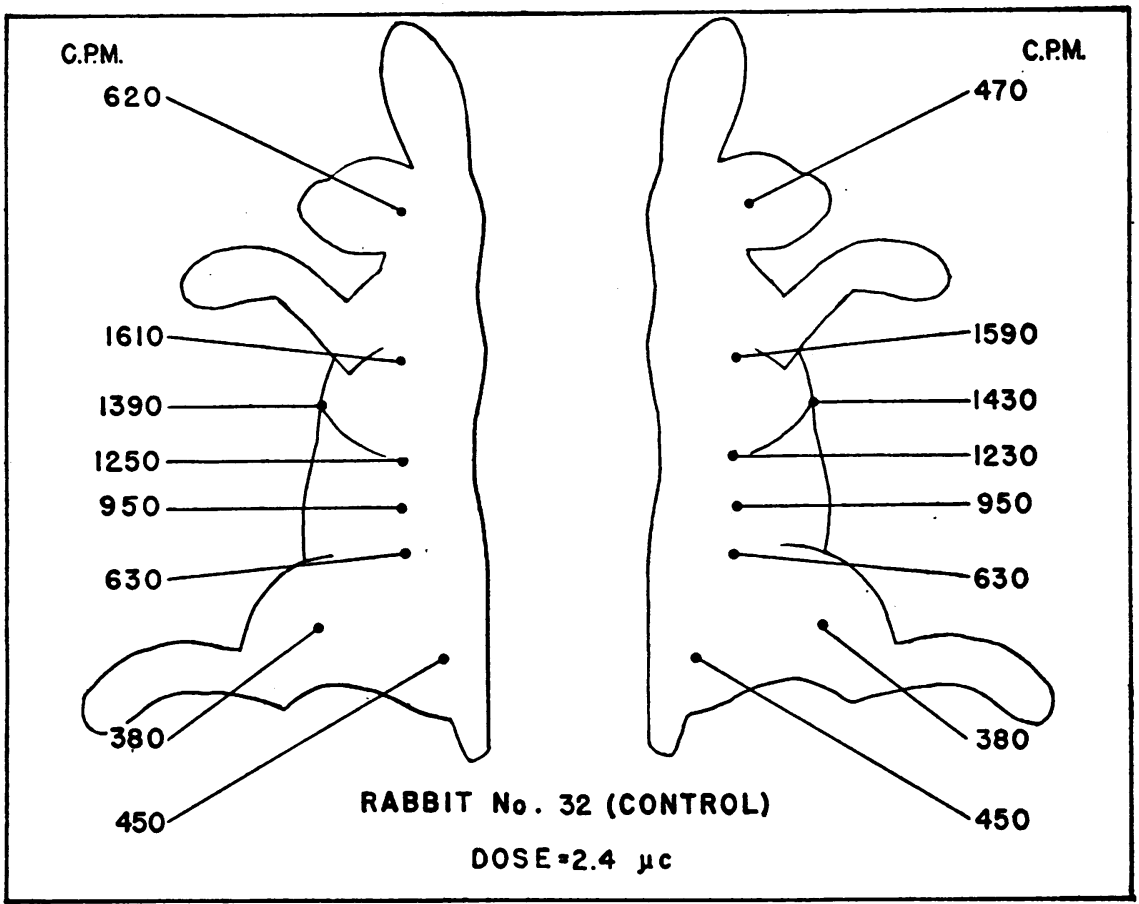

Fig. 4. Distribution of Radionctive Di-Brom Trypan Blue in a Normal Rabbit

Figures are in counts per minute, as registered from the various points shown.

whereas in the rat there is room for only two or three.

The distribution of radioactive di-brom trypan blue in the normal rabbit is shown in Figure 4. The bilateral symmetry of readings from various regions of the body should be noted. This symmetry is consistent within a variation of 10 to 20 per cent. Readings from the two sides of the head may vary to a greater degree than this because it is difficult to place the head of the anaesthetized animal in precisely the same position on the two sides. The same applies to the hind legs, where variations up to 25 per cent between normal sides may be observed.

If the counter is first centered over the upper thorax, in the midline, and then slowly moved down to the pelvis, taking readings each inch or so throughout its course, a set of readings are obtained which may be charted as shown in Figure 5. This indicates that the maximal concentration of dye is found in the region of the xiphoid under which are found the liver, spleen, heart, lungs, and superior splanchnic circulation, all closely grouped and accounting for much of the blood volume of the animal. Insofar as the dye stays in the blood stream, largely, for the first 6 hours after injection, it is to be expected that this area will contain most of the dye. Furthermore, as the dye leaves the circulation, it is taken up by the reticulo-endothelial system (5), and as this same region contains the liver and spleen, it will continue to contain much of the dye even after the dye leaves the blood stream.

It is to be noted in Figure 5 that as the counter is moved down the abdomen of the animal, away from these viscera, the resultant chart is a straight line. That is, the decrease in C.P.M. is proportional to the distance from the xiphoid at which the count is taken.

These three points, then, bilateral symmetry, maximal counts over the xiphoid, and "linearity of decrease" of C.P.M. as the counter is moved toward the pelvis, are the chief normal findings of distribution of the dye in the animal from 1 to 6 hours after it is injected. The absolute values may vary; the pitch or absolute height of the abdominal "line" may vary, but symmetry of distribution, maximal counts over the xiphoid, and a straight line of decrease down the abdomen have been our findings in $\mathbf{5 3}$ observations on $\mathbf{1 5}$ control 


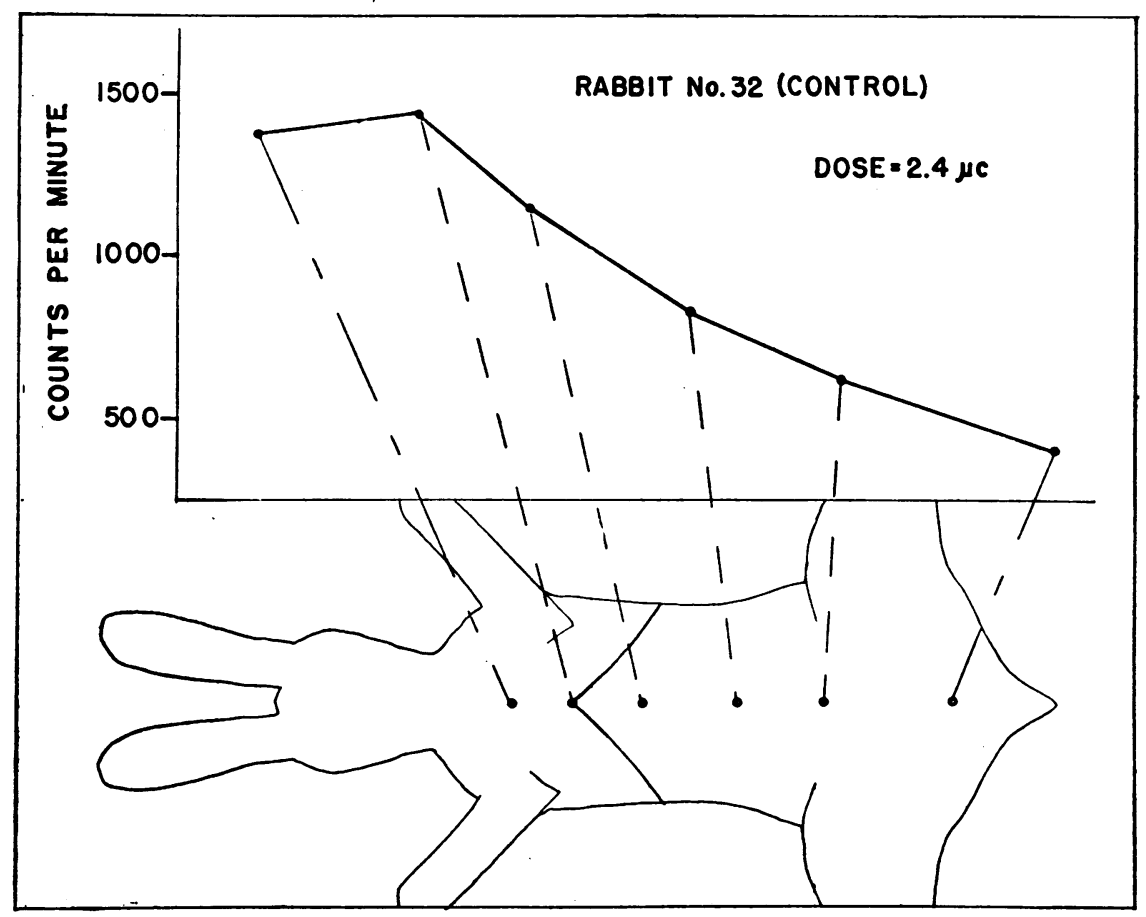

Fig. 5. Chart Showing the Readings Taken from Various Points on the Midline of a Normal Rabbit after the Injection of Radioactive Di-Brom Trypan BLUE

The decline in readings as the counter is moved from the xiphoid to the pelvis approximates a straight line.

animals. In this same group, the average difference in C.P.M. between two normal sides was 10.9 per cent, the mean variation was 22.25 per cent, and the maximum (one instance) was 44.5 per cent.

\section{Results in rabbits with inflammatory lesions}

Our first study was devoted to lesions well away from the large blood-containing viscera in the upper abdomen. These lesions were on the hind legs where the accumulation of radioactivity due to the lesion would be superimposed on a normally low reading.

The findings in a rabbit with a hind-leg lesion are shown in Figure 6 . The inflammatory lesion has produced an increment of 105 per cent more C.P.M. than registered from the contralateral normal leg.

This indicates concentration of the radioactive dye in the lesion to an extent detectable with the counter, and hence permitting diagnostic localization of the lesion by this technique.
This result can be produced with regularity. The average variation between normal sides, in a control group of 6 normal animals in which leg readings were taken, was 6.1 per cent. In an experimental group of 9 animals with leg lesions the average increment produced by inflammation was 91.0 per cent. There were no failures.

When the lesion is in the subcutaneous tissues of the abdominal wall, a different problem presents itself. The accumulation of radioactivity attendent upon the inflammatory process is superimposed on the high concentration of radioactivity normally found in the abdomen. We cannot expect a 90 per cent increment in C.P.M. due to an inflammatory process when that process is small and localized near tissues which normally accumulate a large proportion of the injected radioactive dye. In fact, the increase in C.P.M., due to an inflammatory process on the abdomen, may be only in the range of 10 to 15 per cent over the normal figure for that point on the animal.

However, if we turn our attention to the pattern 
of distribution of the dye-the abdominal "line" -rather than the absolute C.P.M., we find that a significant distortion of that pattern may be produced by an inflammatory process.

It will be recalled that the normal linear decrease in C.P.M. is produced as the counter is moved caudad from the xiphoid (Figure 5). In the presence of an inflammatory process, this linear pattern may be distorted by having a "bump" in it, corresponding in location to the presence of the abscess. Such a distortion may be quite large and gross in character, as shown in Figure 7, or it may be a relatively small distortion, as shown in Figure 8. However, if the distortion of linearity constitutes an increase of 15 per cent or more over the expected figure (indicated by the dotted line), it is considered significant.

The data on inflammatory processes on the abdomen are based on 152 readings taken from 23 animals. In all these, "linearity" or its distortion was studied. In 7 normal animals, all showed normal charts of reading taken from the abdomen. There were no departures from a straight line amounting to 10 per cent or more of the readings. The remaining 16 animals had inflammatory processes. Of these 10 , or 62.5 per cent, showed positive distortion of the linear pattern. Six, or 37.5 per cent, showed essentially straight lines of decrease in C.P.M. as the counter was moved caudad from the xiphoid; in 2 cases, the lines were not straight but the distortion over the inflammation was inconsequential. Of the 6 animals in whom agar and staphylococci had been injected, but which did not accumulate dye to a detectable extent, 3 failed to show evidence of acute reaction to the organisms. There was no edema, heat, redness or evident vasodilatation. The remaining 3 negative animals showed sufficient inflammatory reaction to warrant expectation that they should have concentrated the dye. If we exclude the 3 animals which did not develop a local lesion, the percentage of positive results is 77 per cent for inflammatory processes under the skin of the abdomen.

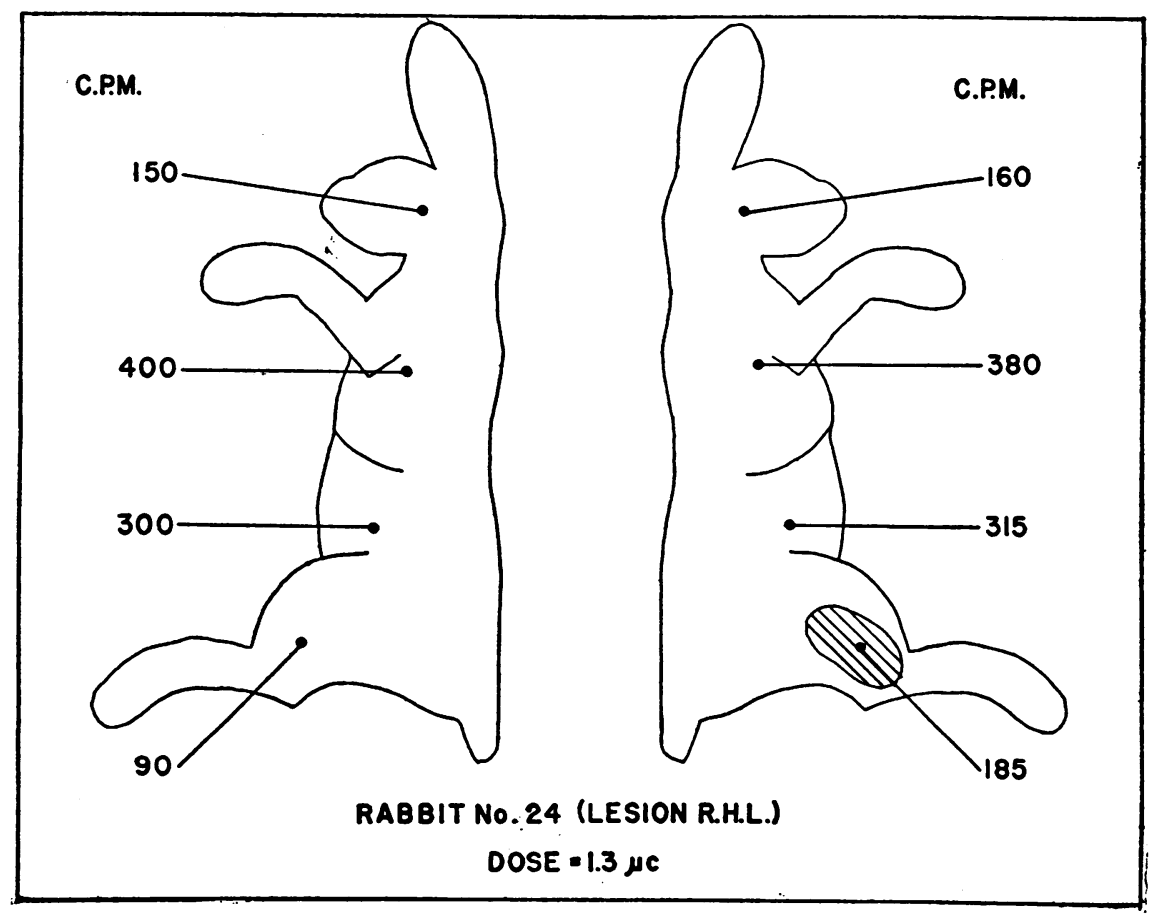

Fig. 6. Findings in a Rabbit with an Inflammatory Process on the Right HIND LEg

The animal has received an injection of $1.3 \mu \mathrm{c}$. of radioactive dye. The figures are in counts per minute from the various points shown. The inflammatory process has accumulated 105 per cent more dye than the control area on the other side. 


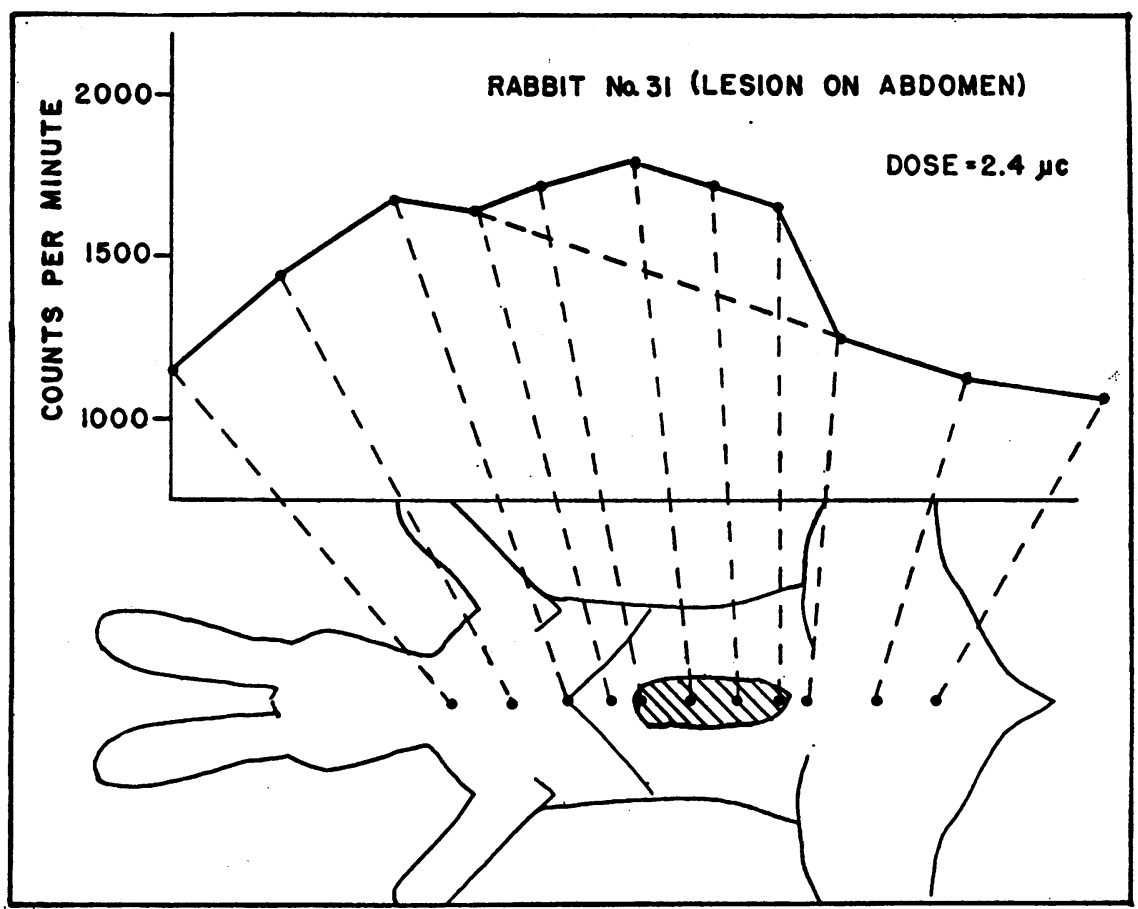

Fig. 7. Chart Showing the Readings Taken from Various Points on the Midline of a Rabit Which has Received Radioactive Dye

There is an inflammatory process in the shaded area. The normally linear decline in readings is distorted by a rise over the inflamed area.

\section{Experiments with bromide ion}

In view of these findings with a dye made radioactive with radio-bromine, it is of interest to investigate the picture presented by the animal which has received an injection of radioactive bromine alone (as sodium bromide).

Weir and Hastings (21) have shown that bromide ion is distributed equally throughout the extracellular fluids. The readings from a normal animal which has received an injection of radiobromide is shown in Figure 9. It will be noted that there is no significant decrease in readings as the counter moves caudad over the abdomen. The legs, furthermore, show somewhat higher readings than is usually found with radioactive dye. The abdominal readings are proportionately low for the magnitude of the leg readings, as compared with dye distribution.

The contrast of this picture with that of the distribution of the colloidal dye reflects the difference in distribution of a substance which is intravascular, from one which is partitioned throughout all the extracellular fluid. The intra- vascular substance shows apparent concentration around the large blood-containing viscera, whereas the ion distributed throughout extracellular fluid shows a distribution more nearly proportional to the simple cross-section of the body at that point. This corroborates the conclusion drawn from the chemistry of the dye, that the "tracer" is being carried through the body by the larger organic molecule of which it is a part, rather than assuming a distribution of its own.

Our results indicate that bromide ion concentrates to a slight extent in inflammatory processes, probably in the edema fluid.

\section{Measurements of tissue radioactivity}

Another approach to this problem is the excision of tissue from the inflamed area, and the study of the amount of radioactivity it has taken up, in contrast to that contained in adjacent normal tissue. To do this, the excised tissue is reduced to an ash in the presence of silver and its radioactivity, as silver bromide, is read directly under the counter. In rabbit No. 31, which gave un- 


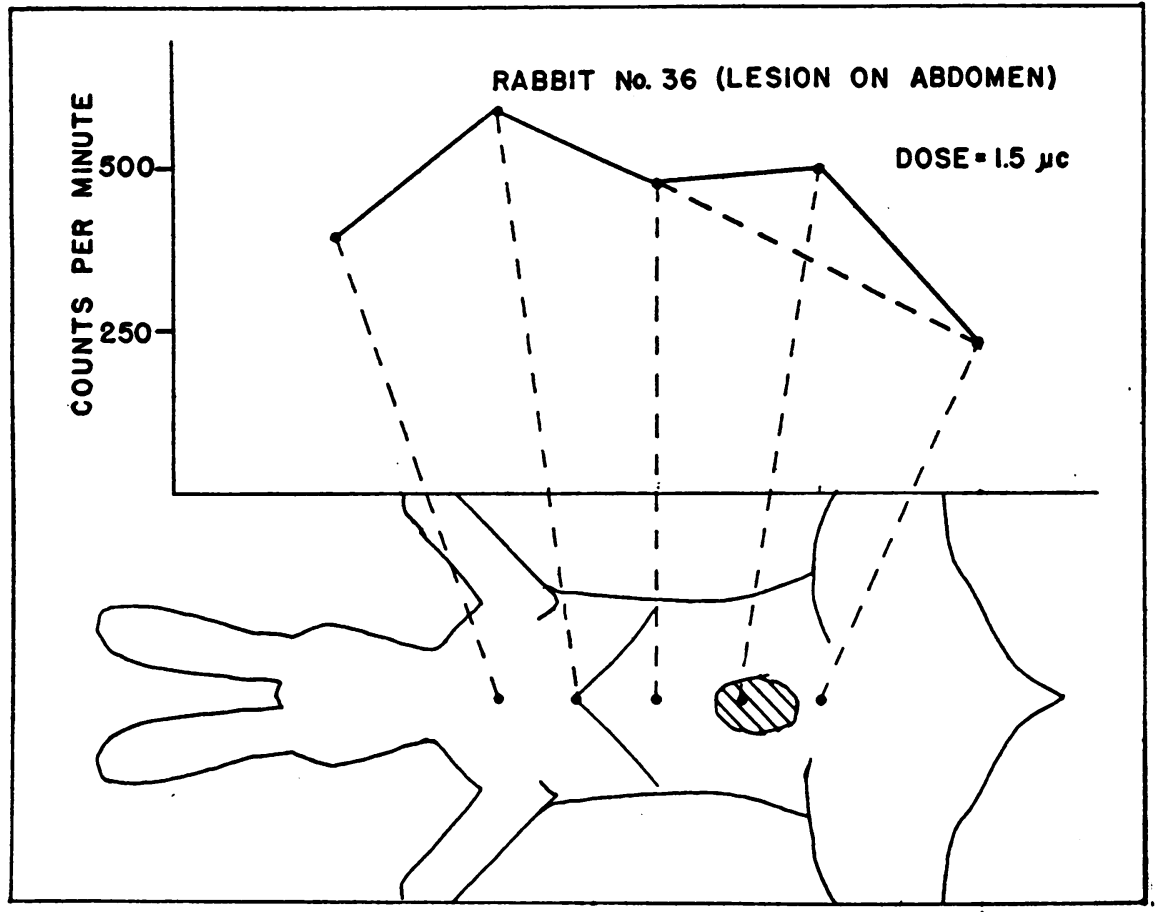

Fig. 8. Chart Showing the Readings Taken from Various Points on the Midline of a Rabbit Which has Received Radioactive Dye

There is an inflammatory process in the shaded area. The normally linear decline in readings has been distorted by the inflammation but to a lesser extent than in the animal shown in Figure 7.

mistakable evidence of the presence of its abscess by the accumulation of radioactive dye (Figure 7), approximately 110 per cent more radioactivity per gram of tissue was found in the area surrounding the inflammatory process than in the neighboring normal tissue. In another animal, in which the original counter readings gave little evidence as to the whereabouts of the abscess, dissection revealed a very quiescent process, and only 40 per cent more radioactive dye per gram of tissue was found in the tissues around the agar nidus, than in adjacent normal tissue. Evidently the animal's resistance to the organism was such as to preclude the appearance of an abscess at the site of injection, and thus to prevent the accumulation there of enough dye to increase the counter reading.

\section{Discussion}

Radioactive di-brom trypan blue gives us a means of diagnosing the location of abscesses on the legs or in the subcutaneous tissues of the abdominal wall of the rabbit. The leg lesions present a fairly simple problem, and so long as there is any inflammation there, enough dye will accumulate to make the lesion evident on the counter. The abdominal lesions, on the other hand, present a less favorable situation, and the results are less gratifying, since in only 77 per cent of the animals could a positive correlation be made of the presence of a lesion and localization of a detectable amount of dye. In the case of the abdominal lesions, the inflammatory process must be quite intense in order to accumulate enough dye to be demonstrable from outside the body.

The failure of some of the animals to concentrate a detectable amount of dye in these lesions is attributable to three factors:

(1) There is a normal variation in the ability of animals to concentrate the dye in inflammatory lesions, as noted by previous workers with dye (9a). 


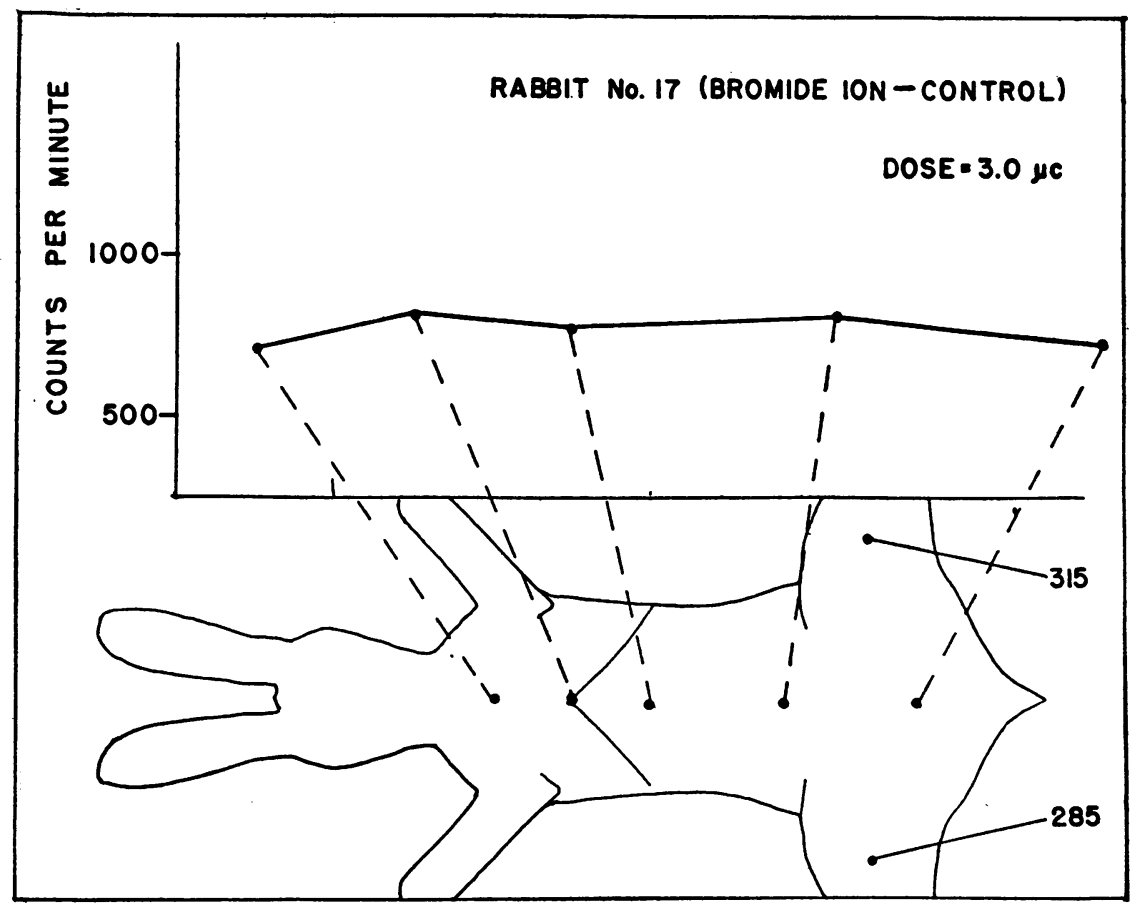

Fig. 9. Chart Showing the Readings Taken from Various Points on the Midline and Legs of a Rabbit Which has Received an Injection of Radioactive Bromine as Sodium Bromide

There is only a slight decrease in readings as the counter moves down the abdomen. The leg readings are relatively higher than those found using radioactive dye.

(2) Variations in virulence of the organism or resistance of the animal may lead to such a mild process that the dye is not accumulated.

(3) This particular dye does not seem to concentrate as consistently in inflammatory lesions as does its non-brominated counterpart, trypan blue.

The first two of these factors are important whenever one attempts to simulate human lesions with staphylococcic abscesses. Virulence and resistance cannot be closely enough controlled to produce unfailing abscess-formation. Properties of the dye may also be an important factor in its failure to accumulate in lesions on some of the animals. As previously pointed out, the molecular weight has been increased, the dye is less soluble in water, and some particulate dye may be filtered out in lungs or liver and thus lost to the inflammatory area. Shortcomings such as these, inherent in the dye itself, can only be overcome by further study of this dye or of other similar dyes such as its isomer, radioactive di-brom Evans blue. Such study is in progress at present.

\section{SUMMARY}

(1) A study of the distribution of radioactive di-brom trypan blue in normal and inflamed animals is described.

(2) This radioactive colloidal dye concentrates in inflammatory lesions to an extent detectable from outside the intact animal with a suitable counter.

(3) Using this radioactive dye, lesions in the periphery of the body were detectable in all cases, whereas abdominal lesions were detectable in 77 per cent of instances.

The authors wish to express their gratitude to Dr. Joseph C. Aub, Dr. Waldo E. Cohn and Dr. Austin M. Brues for their continued interest and valuable advice, and to Prof. Louis F. Fieser for his assistance in regard to the chemistry involved. 


\section{BIBLIOGRAPHY}

i. Goldmann, E. E., Die Äussere und Innere Sektretion des Gesunden und Kranken Organismus im Lichte der "vitale Färbung." Beitr. z. Klin. Chir., 1909, 64, 192. Quoted by Burrows (11).

2. Bowman, F. B., Winternitz, M. C., and Evans, H. M., Ueber die vitale Färbung des Tuberkels. Centralb. f. Bakt., 1912, 65, 403.

3. Kline, R. S., and Winternitz, M. C., Studies upon experimental pneumonia in rabbits. VIII. Intravitam staining in experimental pneumonia and the circulation in the pneumonic lung. J. Exper. Med., 1915, 21, 311.

4. MacCurdy, J. T., and Evans, H. M., Experimentelle Läsionen des Centralnervensystems, untersucht mit Hilfe der vitalen Färbung. Berl. Klin. Wchnschr., 1912, 49, 1695.

5. Evans, H. M., The macrophages of mammals. Am. J. Physiol., 1915, 37, 243.

6. Evans, H. M., and Schulemann, W., The action of vital stains belonging to the benzidine group. Science, 1914, 39, 443.

7. Duran-Reynals, F., A general permeability-increasing effect of a factor from mammalian testicle on blood capillaries. Yale J. Biol. and Med., 1939, 11, 601.

8. Menkin, V., Studies on inflammation. I. Fixation of vital dyes in inflamed areas. J. Exper. Med., 1929, 50, 171.

9. Menkin, V., The Dynamics of Inflammation. Macmillan, New York, 1940.

9a. Menkin, V., Personal communication.

10. Rigdon, R. H., Capillary permeability in the skin of the rabbit. Proc. Soc. Exper. Biol. and Med., 1939, 42, 43.
11. Burrows, H., Some Factors in the Localization of Disease in the Body. Wm. Wood and Co., New York, 1932.

12. Keith, N. M., Rowntree, L. G., and Geraghty, J. T., $A$ method for the determination of plasma and blood volume. Arch. Int. Med., 1915, 16, 547.

13. Gibson, J. G., Jr., Personal communication.

14. Dawson, A. B., Evans, H. M., and Whipple, G. H., Blood volume studies. III. The behaviour of a large series of dyes introduced into the circulating blood. Am. J. Physiol., 1920, 51, 232.

15. Gregerson, M. I., Gibson, J. G., and Stead, E. A., Plasma volume determinations with dyes; errors in colorimetry; use of the blue dye T-1824. Am. J. Physiol., 1935, 113, 54.

16. Strauss, S. F., Neuwelt, F., Rovner, L., and Necheles, H., A new method for the detection of hidden abscesses. Surgery, 1938, 4, 930.

17. Kroll, H. H., Strauss, S. F., and Necheles, H., Concentration and detection of a dye in abscesses. Proc. Soc. Exper. Biol. and Med., 1940, 43, 228.

18. Kroll, H. H., Strauss, S. F., and Necheles, H., Studies on the detection of abscesses and tumors. III. Concentration and detection of a radioactive substance in abscesses. J. Lab. and Clin. Med., 1941, 27, 50.

19. Seaborg, G. T., Artificial radioactivity. Chem. Rev., 1940, 27, 199.

20. Tobin, L. H., and Moore, F. D., Studies with radioactive di-azo dyes. III. The synthesis and properties of radioactive di-brom trypan blue and radioactive di-brom Evans blue. In preparation.

21. Weir, E. G., and Hastings, A. B., The distribution of bromide and chloride in tissues and body fluids. J. Biol. Chem., 1939, 129, 547. 